\title{
Identifying effective guaranteed connections in a multimodal public transport network
}

\author{
Daniel Sparing • Rob M.P. Goverde
}

\begin{abstract}
Minimizing transfer waiting time is important in making public transport networks more attractive. A guaranteed transfer, with the departing vehicle waiting on moderately delayed arriving vehicles at a transfer node, is an effective way to reduce waiting times at transfers between low frequency public transport lines. This secondary train delay comes at the cost of a new delay for non-transferring passengers. The method described in this paper, based on max-plus algebra, classifies potential connections based on their feasibility for given initial delays, in order to help operational decisions on-line and to assist public transport companies off-line in identifying transfers vulnerable to delays. A case study shows the applicability of the approach for a real-life multimodal network.
\end{abstract}

Keywords Guaranteed transfers · Max-plus algebra · Multimodal timetable

\section{Introduction}

Public transport users prefer direct, short, high-frequency services in order to minimize travel time, waiting time and inconvenience. On the other hand, it is neither possible nor efficient to provide direct connections between all origin and destination pairs. Similarly, high-frequency services are not justified by demand on all public transport routes. Therefore it is inevitable for any public transport network to include some transfer connections between low frequency lines.

Daniel Sparing

Delft University of Technology

P.O. Box 5048, 2600 GA Delft, The Netherlands

Tel.: +31-15-27-84914

Fax: +31-15-27-83179

E-mail: d.sparing@tudelft.nl

Rob M.P. Goverde

Delft University of Technology

E-mail: r.m.p.goverde@tudelft.nl 
A measure to tackle the issue of long transfer waiting times is timed transfers: a timetable which synchronizes arrival and departure times of different lines in order to make the waiting time significantly lower than the headway. However, in case of arrival delays, timed transfers can actually result in a higher overall waiting time than in the case of uncoordinated timetables. Therefore, a timed transfer can additionally be guaranteed when a departing vehicle waits for moderately delayed arriving vehicles. A guaranteed transfer significantly improves the travel experience of transferring passengers while causing only relatively mild delays for other passengers. Nonetheless, there is a cost to guaranteed transfers and this cost also depends on the scheduled slack times in the timetable of the departing vehicle; in other words, on how fast the departure delay can be absorbed.

This connection management has already received significant attention in literature, with different modelling assumptions and target functions. Knoppers and Muller (1995) examine when timed transfers are beneficial with respect to frequency and reliability conditions. Goverde (1998) focuses on train networks with predefined connections and makes a distinction between waiting time in vehicles and on platforms. Heidergott and De Vries (2001), also describe interconnected train networks and introduce some heuristics to reduce the solution space created by multiple connections. Ginkel and Schöbel (2007) use a hybrid criteria of passenger volumes for a missed connection and vehicle delay for a maintained connection. For a further review of connection management literature, we refer to Ginkel and Schöbel (2007).

This paper uses efficient delay propagation methods based on the max-plus algebra approach to identify connections at risk for a given delay scenario. By defining a subset of the full public transport network as the controllable component, the scope of the calculations for optimal control is significantly smaller than the whole network where initial delays are taken into account. An objective function based on passenger delays caused by both missed connections and arrival delays is used.

The paper is organised as the following. Section 2 describes the mathematical model for periodic public transport networks and the max-plus algebra representation. Section 3 explains the approaches to reduce the solution space via defining significant connections and splitting the network to controllable and uncontrollable parts. Section 4 describes a case study based on a real-life network and Section 5 concludes the paper.

\section{The periodic public transport network model}

A public transport network operating according to a periodic schedule can be modelled as a discrete-event dynamic system (Goverde 2010) as follows. Let $T$ be the timetable period, often $T=60 \mathrm{~min}$ and $i$ a certain periodic timetable event such as a departure, arrival or passage at a station. Then $x_{i}(k)$ is the event time of event $i$ scheduled in period $k$. Hence, if $k=0$ represents the initial period $[0, T)$, then $k=1$ represents the next period 
$[T, 2 T)$, and in general $x_{i}(k)$ is scheduled in period $[k T,(k+1) T)$. Let $D, A$ and $P$ denote a departure, arrival, and passing event, respectively. Then an event $i$ can be identified by a triple of attributes $i=\left(d_{i}^{0}, S_{i}, L_{i}, T_{i}\right)$, where $d_{i}^{0}$ is the initial scheduled event time, $S_{i}$ is the station of the event, $L_{i}$ the line, and $T_{i} \in\{A, D, P\}$ is the event type. Note that $d_{i}$ denotes either a scheduled departure, arrival or passage time.

\subsection{Schedule constraints}

The public transport timetable defines scheduled event times $d_{i}(k)$ for the departure, arrival and passing events $i$. While early arrival and passing events are usually allowed, early departures are typically forbidden in most public transport networks. This results in the constraints

$$
x_{i}(k) \geq d_{i}(k), \quad i \in\left\{i: T_{i}=D\right\} .
$$

Recall that $\left\{i: T_{i}=D\right\}$ is the set of departure events.

\subsection{Precedence constraints}

The successive events on a given vehicle journey are connected by activities with given minimum process times like minimum running, dwell, and layover times. Moreover, infrastructure restrictions - especially on railway infrastructure - may imply that events of different lines using the same piece of infrastructure can only take place with sufficient minimum headway time elapsed between them, and moreover, the timetable fixes an order of the vehicles. Such constraints can be following trains on the same railway track, merging or crossing trains at a railway junction, or trains of opposite direction passing at loops on single track lines.

Both journey and infrastructure constraints can be defined between any departure, arrival, or passing event as a preceding constraint of the form

$$
x_{i}(k) \geq a_{i j}(k)+x_{j}\left(k-\mu_{i j}\right),
$$

where $a_{i j}(k) \geq 0$ is the minimum process time from event $j$ to $i$ in period $k$, and $\mu_{i j} \in \mathbb{N}_{0}$ is the period shift, meaning that event $i$ is scheduled $\mu_{i j}$ periods later than $j$, with $\mu_{i j}=0$ thus implying that events $i$ and $j$ are in the same period. Based on the scheduled event times, $\mu_{i j}$ can be calculated as (Goverde 2007)

$$
\mu_{i j}=\frac{a_{i j}^{0}+d_{j}^{0}-d_{i}^{0}}{T}
$$

where $a_{i j}^{0}$ is the scheduled process time given in full minutes and $d_{i}^{0}, d_{j}^{0}$ are the scheduled event times in the initial period. Note that $a_{i j}^{0}>a_{i j}(k)$ in case of a positive time reserve for the given process. For any given network it is possible to transform the model, by adding dummy events, so that $\mu_{i j} \in\{0,1\}$ for all $(j, i)$ (Goverde 2005). 


\subsection{Controllable connections}

Connection constraints describe the interdependency of an arrival event of one line and a departure event of another line because of a shared resource (vehicle or crew) or because of a guaranteed passenger transfer. Vehicle or crew constraints are hard connections that cannot be relaxed, so they are also modelled as a precedence constraint (1) from an arrival to a departure event. On the other hand, some passenger transfers can be seen as 'soft' connections, or controllable, that can be activated or not based on the actual vehicle delays.

These soft connections can be modelled using the decision variables $\delta_{i j}(k) \in$ $\{0,-\infty\}$, which mean that the controllable connection from event $j\left(k-\mu_{i j}\right)$ to $i(k)$ is active if $\delta_{i j}(k)=0$ and inactive if $\delta_{i j}(k)=-\infty$. This is modelled as

$$
x_{i}(k) \geq a_{i j}(k)+\delta_{i j}(k)+x_{j}\left(k-\mu_{i j}\right) .
$$

Note that an inactive soft connection is broken while an active connection implies a guaranteed transfer.

We assume that a list $C$ of controllable connections is available. Note that a controllable connection is a pair $(j, i)$ of an arrival event $j$ and a departure event $i$ with a given minimum transfer time $a_{i j} \geq 0$ and a decision variable $\delta_{i j} \in\{-\infty, 0\}$. Not all arrival/departure pairs of different lines at a given station are generally defined as a connection. In particular, a minimum transfer time must be available in the timetable to enable the transfer in punctual operations and moreover a maximum waiting time applies up to which we still speak of a (controllable) connection. If a a list of possible connections is not available then we may generate a list from the timetable. Assume that the minimum transfer time $t_{\min }$ for a given arrival and departure platform pair is given, and that the maximum acceptable waiting time $w_{\max }$ is defined. Then, the set of possible soft connections is given as

$C=\left\{(j, i) \mid T_{j}=A, T_{i}=D, S_{j}=S_{i}, L_{j} \neq L_{i},\left(d_{i}^{0}-d_{j}^{0}-t_{\min }\right) \quad \bmod T<w_{\max }\right\}$.

In case of such a generated connection list, however, extra care has to be taken to filter out potential connections which are not attractive to the passengers. This is possible by taking into account passenger flows.

\subsection{Max-plus algebra representation}

Discrete event systems as described above can be formulated and analysed effectively in max-plus algebra (Heidergott et al 2005). In particular, maxplus models have been applied successfully in the evaluation of periodic railway timetables (Braker 1993; Goverde 2007).

Max-plus algebra is an algebraic structure defined on $\mathbb{R}_{\max }:=\mathbb{R} \cup\{-\infty\}$ with the max operator and addition instead of addition and multiplication in conventional algebra, respectively. This structure satisfies the properties of 
an idempotent semiring, see e.g. Heidergott et al (2005). That is, max-plus addition and multiplication are defined for $a, b \in \mathbb{R}_{\max }$ as

$$
a \oplus b:=\max (a, b) \text { and } a \otimes b:=a+b .
$$

Matrix addition $\oplus$ and matrix multiplication $\otimes$ are defined analogous to conventional algebra, i.e., for matrices $A, B \in \mathbb{R}_{\max }^{n \times n}$

$$
\begin{aligned}
& (A \oplus B)_{i j}:=a_{i j} \oplus b_{i j}=\max \left(a_{i j}, b_{i j}\right) \\
& (A \otimes B)_{i j}:=\bigoplus_{k=1}^{n}\left(a_{i k} \otimes b_{k j}\right)=\max _{k=1, \ldots, n}\left(a_{i k}+b_{k j}\right) .
\end{aligned}
$$

The precedence and controllable connection constraints above can be rewritten using max-plus algebra notation as follows. Let $\Pi$ denote the set of all precedence constraints, including hard connections, and $C$ the set of all controllable connections. Then the model becomes in max-plus algebra

$$
x_{i}(k)=\bigoplus_{(j, i) \in \Pi}\left(a_{i j} \otimes x_{j}\left(k-\mu_{i j}\right)\right) \bigoplus_{(l, i) \in C}\left(a_{i l} \otimes \delta_{i l}(k) \otimes x_{l}\left(k-\mu_{i l}\right)\right) \oplus d_{i}(k) .
$$

This model can be written in vector notation as follows. Collect all event times $x_{i}(k)$ in the event time vector $x(k)=\left(x_{1}(k), \ldots, x_{n}(k)\right)^{\prime} \in \mathbb{R}^{n}$, and define the (uncontrollable) matrices $A_{0}, A_{1} \in \mathbb{R}_{\max }^{n \times n}$ as

$$
\left(A_{l}(k)\right)_{i j}= \begin{cases}a_{i j}(k) & \text { if }(j, i) \in \Pi \text { and } l=\mu_{i j} \\ -\infty & \text { otherwise }\end{cases}
$$

and the controllable matrices $B_{0}, B_{1} \in \mathbb{R}_{\max }^{n \times n}$ as

$$
\left(B_{l}(u(k), k)\right)_{i j}= \begin{cases}a_{i j}(k)+\delta_{i j}(k) & \text { if }(j, i) \in C \text { and } l=\mu_{i j} \\ -\infty & \text { otherwise }\end{cases}
$$

The vector $u(k)$ is the control vector in period $k$ which determines which of the connections in period $k$ are broken and which are not, i.e., $u(k)$ sets $\delta_{i j}(k)=-\infty$ for all connections $(i, j) \in C$ that are broken and $\delta_{i j}(k)=0$ otherwise. Then the model can be written in matrix notation as

$x(k)=A_{0}(k) x(k) \oplus A_{1}(k) x(k-1) \oplus B_{0}(u(k), k) x(k) \oplus B_{1}(u(k), k) x(k-1) \oplus d(k)$.

Alternatively, this can be expressed as

$$
x(k)=\left[A_{0}(k) \oplus B_{0}(u(k), k)\right] x(k) \oplus\left[A_{1}(k) \oplus B_{1}(u(k), k)\right] x(k-1) \oplus d(k) .
$$

The model (2) can now be used to find the delay propagation from any given time point for any given past delays (Goverde 2010). Without loss of generality, we may assume a given initial condition $x(0)=x_{0}$, where $x_{0}=d(0)+z(0)$ with $z(0) \geq 0$ the vector of delays in the initial period. From this given initial condition, the event time estimates $x(k)$ can now be calculated for any $k \geq 1$ up to a suitable time horizon $K \geq 1$. In particular, a lower bound of all 
(secondary) delays can be obtained by braking all controllable connections, i.e., $\delta_{i j}=-\infty$ for all $(j, i) \in C$, or equivalently, $B_{0}(u(k), k)$ and $B_{1}(u(k), k)$ having all entries equal to $-\infty$. Then (2) reduces to

$$
x(k)=A_{0}(k) x(k) \oplus A_{1}(k) x(k-1) \oplus d(k), \quad x(0)=x_{0} .
$$

By guaranteeing any additional connection $(j, i) \in C$ the total train delay may increase, but the passenger waiting time might decrease depending on the transferring and onboard passenger volumes and the amount of delay recovery.

\subsection{Passenger delay estimations}

Modelling or forecasting passenger flows for disturbed operations is difficult, especially taking into account the mode choice which is also affected, see Van Eck (2011) for a multimodal traffic model taking into account simultaneous mode choice. On the other hand, simply counting the number of maintained and broken connections is not sufficient as an objective since the demand and attractiveness of different connections can vary significantly. Therefore, at least a rudimentary model for passenger demand is required.

Assume that an estimate of the number of boarding and alighting passengers is known for every stop of every service. Denote by $w_{i}(k)$ the number of boarding or alighting passengers in period $k$, depending on the event type $T_{i}$, i.e., $w_{i}(k)$ is the number of alighting passengers for $\left\{i: T_{i}=A\right\}$ and the number of boarding passengers for $\left\{i: T_{i}=D\right\}$, while by convention $w_{i}(k)=0$ for passage events $\left\{i: T_{i}=P\right\}$. Furthermore, for each controllable connection $(j, i) \in C$ let $w_{i j}(k)$ be the number of transferring passengers from arrival event $j$ to departure event $i$ in period $k$. These passenger count estimates can be provided by previous passenger counts or traffic flow models. See Hilderink et al (2010) for a traffic flow model that models public transport passenger flows at this detail.

If detailed passenger counts are not available or not available over diffferent periods over a day, then we may resort to fixed weights $w_{i}$ and $w_{i j}$ measuring the relative importance of stops and connections. Such passenger counts allow for a reasonable comparison of total passenger delays in case of maintained or broken connections. In case of a maintained connection with a delayed departure, the total arrival passenger delay on the given line is the sum of vehicle arrival delays weighted by the number of alighting passengers per stop. The total arrival delay in the network is given by

$$
\sum_{k=1}^{K} \sum_{j \in\left\{j: T_{j}=A\right\}} w_{j}(k) \cdot \max \left(0, x_{j}(k)-d_{j}(k)\right),
$$

where the maximum of delay and zero is required so that early arrivals do not count. Moreover, the delay due to the missed connections can be estimated as

$$
\sum_{k=1}^{K} \sum_{(j, i) \in M(k)} w_{i j}(k) c_{j},
$$


with $c_{j}$ the cycle time of line $i$ and $M(k)$ the set of missed connections in period $k$ defined as

$$
M(k)=\left\{(j, i) \in C: x_{i}(k)-x_{j}\left(k-\mu_{i j}\right)-a_{i j}<0\right\}
$$

These delays can be compared for different connection controls $u(k)$.

\subsection{The optimization model}

The goal of the optimization model is to find the optimal decision parameters $\delta_{i j}(k)$ for each controllable connection $(j, i)$ and period $k \geq 1$ so that the estimated total passenger delay caused by either maintaining or braking a controllable connection is minimal. Input parameters are the line cycle times $c_{j}$, the passenger weight $w_{i}(k)$ for each arrival event $i$, and the transfer weight $w_{i j}(k)$ for each connection $(j, i) \in C$, as well as the initial condition including delays $x_{0}$, and a time horizon $K \in \mathbb{N}$. Then the optimization problem becomes

$$
\min \sum_{k=1}^{K}\left(\sum_{j \in\left\{j: T_{j}=A\right\}} w_{j}(k) \cdot \max \left(0, x_{j}(k)-d_{j}(k)\right)+\sum_{(j, i) \in M(k)} w_{i j}(k) c_{j}\right)
$$

subject to

$$
\begin{array}{ll}
x_{i}(k) \geq d_{i}(k) & i \in\left\{i: T_{i}=D\right\}, k=1 \ldots, K \\
x_{i}(k) \geq a_{i j}(k)+x_{j}\left(k-\mu_{i j}\right) & (j, i) \in \Pi, k=1 \ldots, K \\
x_{i}(k) \geq a_{i j}(k)+\delta_{i j}(k)+x_{j}\left(k-\mu_{i j}\right) & (j, i) \in C, k=1 \ldots, K \\
\delta_{i j}(k) \in\{-\infty, 0\} & (j, i) \in C, k=1 \ldots, K \\
x(0)=x_{0}, &
\end{array}
$$

where $M(k)$ is the set of missed connections as defined in (4).

The problem is solved using the max-plus interpretation where the delay propagation can be computed very quickly. The constraint set of the above optimization problem can therefore be formulated equivalently as

$$
\begin{aligned}
& \begin{aligned}
x(k)= & A_{0}(k) x(k) \oplus A_{1}(k) x(k-1) \oplus \ldots \\
& B_{0}(u(k), k) x(k) \oplus B_{1}(u(k), k) x(k-1) \oplus d(k) \quad k=1, \ldots, K \\
x(0)= & x_{0},
\end{aligned}
\end{aligned}
$$

where $u(k) \in\{-\infty, 0\}^{|C|}$ is a $|C|$-dimensional control vector encoding the connection decisions. Note that $|C|$ denotes the size of $C$, i.e., the number of controllable connections. 


\section{Solution approach}

The solution to the optimization problem may be found using a standard mixed-integer programming solver. For problems with many controllable connections we may explore the (max-plus) structure of the problem and use a branch-and-bound procedure to find the optimal combination of decision variables. In both cases, reducing tthe dimension of the problem will help solving it more quickly. In this section we give two preprocessing steps that can be used for this aim.

\subsection{Identifying significant connections}

The decision variables are the 'binary' variables $\delta_{i j}(k)$ corresponding to the controllable connections. In a preprocessing step we may reduce this set to only those connections that are significant to the optimization problem. For this we look at the output of the initial delay propagation for the given initial delay scenario and all connections broken. Then the controllable connections can be categorized depending on the initial delay scenario and a maximum allowable departure delay $d_{\max }$ as follows.

- Automatically maintained connections: those arrival-departure pairs where a transfer is possible despite the delays, without further delay of the departure vehicle.

- Significant connections: Those connections that are maintained by delaying the departing vehicle by at most $d_{\max }$.

- Automatically broken connections: Those connections that must be cancelled otherwise the departing vehicle will be delayed by more than $d_{\max } \geq$ 0 .

The set of significant connections $C_{s}$ is thus defined as

$$
C_{s}=\left\{(j, i, k) \in C: 0 \leq x_{i}(k)-a_{i j}(k)-x_{j}\left(k-\mu_{i j}\right) \leq d_{\max }, k=1, \ldots, K\right\},
$$

where the event times $x(k)$ are computed using the delay propagation model (3) with all connections broken. Note that $C_{s}$ contains the significant controllable connections for each period separately. The parameter $d_{\max }$ is called the synchronization margin in Knoppers and Muller (1995). In general, these synchronization control margins may be different for different connections (Goverde 1998). However, in our model it is just a fixed upper bound on the allowed delay, while the outcome of the optimization will determine how long a connecting vehicle will wait on a delay feeder train. For instance, a departing vehicle with ample running time supplement and buffer time ahead may wait longer than a departing vehicle with a tight schedule or a capacity bottle neck nearby.

Hence, a shortlist of significant connections can be obtained rapidly from combining the delay propagation algorithm with a (generated) controllable connection list and using appropriate thresholds. In real-time online usage, 
the significant connections can be retrieved given the actual delays, thus helping drivers and controllers focus on those transfers where decisions have to be made. In off-line timetable evaluation usage, the significant connections, for given realistic delay scenarios, represent the most vulnerable transfers. A timetable planner may then try to modify the timetable to improve the reliability of these transfers. The latter topic can also be done in an optimization context which is another current research topic.

\subsection{The controllable subnetwork}

Another way to decrease the problem size is a partitioning of the network in a controllable and an uncontrollable subnetwork. The controllable subnetwork is a set of lines on which dispatching actions can be made, such as delaying a departure. The uncontrollable subnetwork consists of the remaining lines on which the expected user of the decision support model has no influence. Typically, no vehicle or crew member operates on both subnetworks, while passenger transfers can be defined between the two subnetworks.

As an illustrative example, the controllable subnetwork can be a bus network controlled by a bus dispatcher, while the uncontrollable subnetwork is a train network. In this case, it is realistic to assume that the dispatchers of the bus company have influence on the bus network but not on the train network.

The advantage of this separation is that the optimization problem only has to take the controllable subnetwork into account. Only the initial delay propagation computation with all controllable connections broken must be run for the whole network to find the delays at the boundaries of the controllable network. In the next step, the effect of the different combinations of broken or maintained controllable connections can be evaluated much faster by only calculating delays on the smaller, controllable subnetwork.

\section{Case study}

\subsection{Example network}

The example network consists of a regional bus network of 4 lines in the West of the Netherlands as listed in Table 1 and the Dutch national railway network timetable which have several stations in common, see Figure 1. In this case study, the bus network will be the controllable subnetwork, while the train network is the external, non-controllable subnetwork. The timetables, minimum process times and passenger load values are obtained from different sources and are based on different years between 2004 and 2011. As accurate measured or modelled passenger count data was not available, example passenger flow values were generated for testing reasons. Therefore, the goal of the case study network is not to reflect a real life situation on a given day perfectly, but to provide an example of realistic size and complexity, see Table 2. 


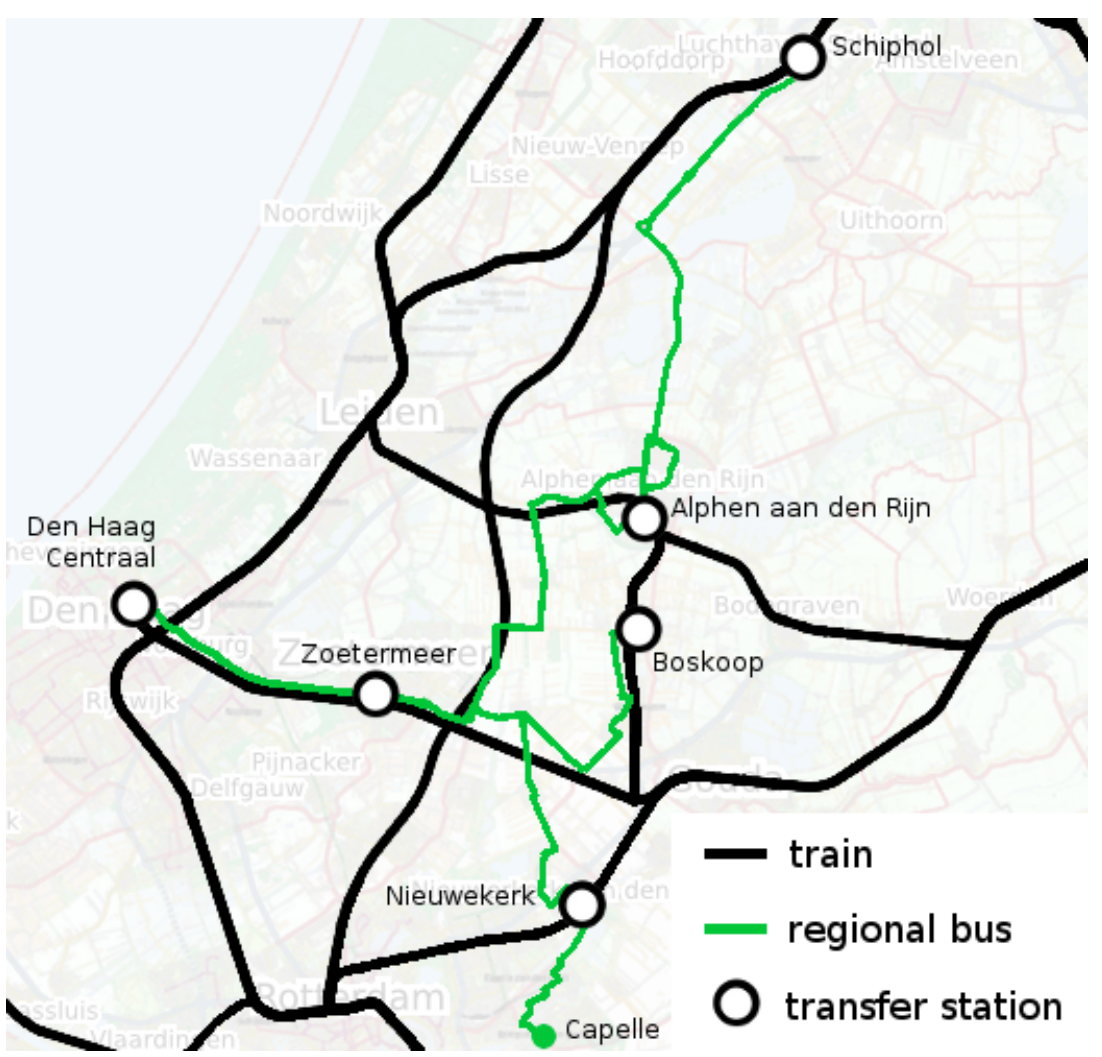

Fig. 1 Network of four bus lines and connecting train lines in the Netherlands

Table 1 List of bus lines

\begin{tabular}{llll}
\hline line & from & to & Cycle time \\
\hline 370 & Schiphol & Alphen aan den Rijn & $15 \mathrm{~min}$ \\
380 & Alphen aan den Rijn & Den Haag Centraal & $30 \mathrm{~min}$ \\
382 & Boskoop & Den Haag Centraal & $60 \mathrm{~min}$ \\
383 & Capelle a/d IJssel & Den Haag Centraal & $60 \mathrm{~min}$ \\
\hline
\end{tabular}

Table 2 Characteristics of the example controllable subnetwork

\begin{tabular}{lc}
\hline Vehicle journeys & $16 /$ hour \\
Stops & 57 \\
Transfer stations & 6 \\
Events & 440 \\
Controllable connections & 95 \\
\hline
\end{tabular}


Table 3 Transfer stations

\begin{tabular}{lc}
\hline station & transfer time (min) \\
\hline Boskoop & 4 \\
Alphen aan den Rijn & 5 \\
Schiphol & 4 \\
Den Haag Centraal & 5 \\
Nieuwekerk a/d IJssel & 5 \\
\hline
\end{tabular}

\subsection{Candidate transfers}

We assume that initially no guaranteed connections are offered between the bus and the train network, i.e., no transfer constraints are defined. We therefore define a list of candidate transfers between arriving trains and departing buses.

As a first step, we expect that there is a list of stop pairs available between bus stops and train stations between which a transfer is physically possible in reasonable time, as well as the estimated minimum transfer time $t_{l}$ required at station $S_{l}$ between alighting from a train to boarding a bus (Table 3).

Furthermore, we define $w_{\max }=6 \mathrm{~min}$ as the maximum acceptable waiting time for a connection. Therefore the connection list $C$ includes all $(j, i)$ arrivaldeparture pairs at transfer station $S_{l}$, with minimum transfer time $t_{l}$, where $t_{l} \leq d_{i}-d_{j} \bmod T \leq t_{l}+w_{\max }$.

\subsection{Initial delay scenario}

To investigate a concrete network status including delay, assume that the delays are known over a full timetable period $k=0$. Without loss of generality, we assume that the moment for the following calculations is the end of period $k=0$ : initial delays within period $k=0$ are known, while consecutive delays in the successive periods $k \geq 1$ can be calculated using the max-plus model, taking into account different dispatching actions. For our calculation purposes, a random initial delay vector is calculated, based on a delay probability $p=$ $10 \%$ for each departure event and a uniform delay distribution between 0 and 10 minutes.

\subsection{Significant connections}

The set of significant connections $C_{s}$ is determined using $d_{\max }=4$ and 20 different delay scenarios generated as described in Section 4.3. Figure 2 shows the classification of connections in the first period. The results show that although there are 95 controllable connections in the model, only about $10-$ $30 \%$ of them are significant and require more attention.

Another insight that can be gained from such results, assuming that not random, but realized or simulated delay distributions are used, is a statistic of how often a given connection becomes significant or broken beyond the maximum departure delay limit. Figure 3 shows for each connection, how often it 


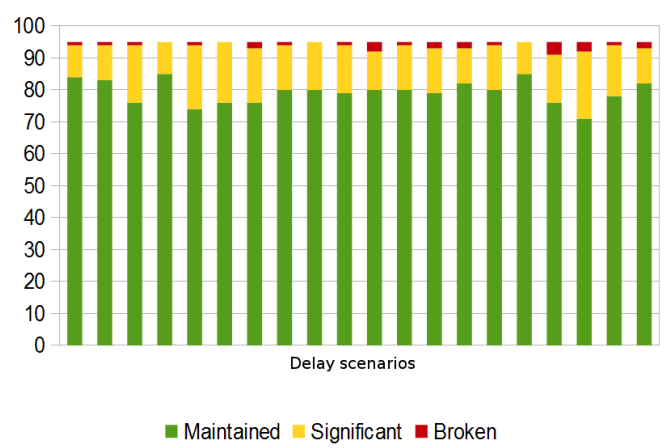

Fig. 2 Connection classifications for the 20 random initial delay scenarios

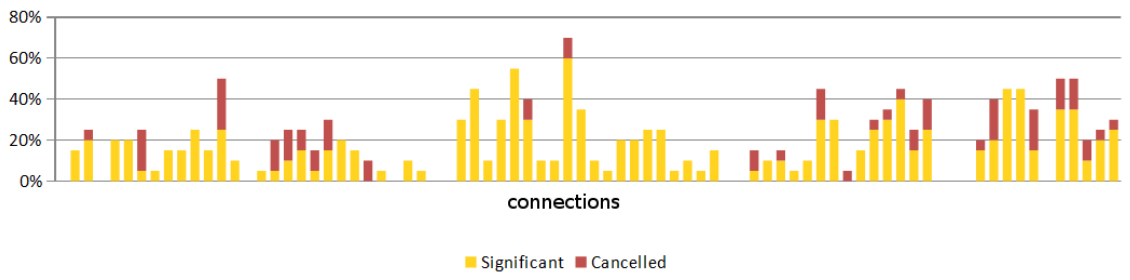

Fig. 3 Histogram of significant connections

becomes significant or automatically broken over the same series of 20 delay scenarios. A connection which would be broken exactly once for any delay scenario would have a value of $100 \%$. Such results, together with information on the demand, help timetable planners and operating staff to identify vulnerable transfers requiring more attention.

\subsection{Optimal connection control}

For a given delay scenario and set of significant connections, the solution of the optimization problem (Section 2.6) returns the combination of maintained or cancelled transfers with the lowest total passenger delay. In our example, a set of initial delays results in 17 significant and 2 automatically broken connections. Using generated example passenger count data, Table 4 shows the total passenger arrival delay and the total passenger transfer delay in case of all significant connections cancelled or maintained. Furthermore, Figure 4 and Figure 5 shows the vehicle and passenger delays, respectively, in case of all connections cancelled. It is visible that even in case of moderate vehicle delays, passenger delays can be significant because of missed transfers.

In case of all connections maintained, the total passenger transfer delay is significantly smaller, as the passengers of the maintained connections do 
Table 4 Optimization results

\begin{tabular}{lrrr}
\hline control strategy & $\begin{array}{r}\text { arrival delay } \\
\text { (hh:mm:ss) }\end{array}$ & $\begin{array}{r}\text { transfer delay } \\
\text { (hh:mm:ss) }\end{array}$ & $\begin{array}{r}\text { total delay } \\
\text { (hh:mm:ss) }\end{array}$ \\
\hline all connections cancelled & $51: 14: 13$ & $83: 15: 00$ & $134: 29: 13$ \\
all connections maintained & $65: 36: 03$ & $06: 00: 00$ & $71: 36: 03$ \\
optimal control & $58: 07: 51$ & $07: 15: 00$ & $65: 22: 51$ \\
\hline
\end{tabular}

not experience transfer delay anymore. This comes at the relatively lower cost or increased arrival delays. Note that there are still arrival delays for all significant connections cancelled, because of the initial delays within the controllable network; while there is some transfer delay even for all significant connections maintained, due to the two automatically broken connnections.

The optimal control strategy consists of a mixture of some 6 significant connections maintained and the other 11 cancelled. The final row in Table 4 shows the this control strategy, with the lowest total delay. Figure 6 shows passenger delay for the optimal control, where the extent of total passenger delay is reduced as compared to the first case without guaranteed connections (Figure 5).

\section{Conclusion}

The presented numerical model can assess the influence of guaranteed transfers on timetable stability in case of delays. The use of max-plus algebra results in very short computational times, even on real-life networks. This approach is suitable to support both long-term timetable coordination between different operators and real-time analysis of delay propagation given a set of actual delays.

Acknowledgements This work is part of the research programme Sustainable Accessibility of the Randstad, which is financed by the Netherlands Organisation for Scientific Research (NWO). The authors also thank the 9292 REISinformatiegroep BV for making public transport timetable data available for this research.

\section{References}

Braker JG (1993) Algorithms and Applications in Timed Discrete Event Systems. Phd thesis, TU Delft

Ginkel A, Schöbel A (2007) To Wait or Not to Wait? The Bicriteria Delay Management Problem in Public Transportation. Transportation Science 41(4):527-538, DOI $10.1287 /$ trsc. 1070.0212

Goverde RMP (1998) Synchronization Control of Scheduled Train Services to Minimize Passenger Waiting Times. In: Proceedings 4th TRAIL Year Congress, Part 2, TRAIL Research School, Delft

Goverde RMP (2005) Punctuality of Railway Operations and Timetable Stability Analysis. Phd thesis, TU Delft

Goverde RMP (2007) Railway timetable stability analysis using max-plus system theory. Transportation Research Part B: Methodological 41(2):179-201, DOI 10.1016/j.trb.2006.02.003 
Goverde RMP (2010) A delay propagation algorithm for large-scale railway traffic networks. Transportation Research Part C: Emerging Technologies 18(3):269-287, DOI 10.1016/j.trc.2010.01.002

Heidergott B, De Vries R (2001) Towards a (Max,+) Control Theory for Public Transportation Networks. Discrete Event Dynamic Systems: Theory and Applications 11:371-398

Heidergott B, Olsder GJ, van der Woude J (2005) Max Plus at Work: Modeling and Analysis of Synchronized Systems: A Course on Max-Plus Algebra and Its Applications. Princeton University Press

Hilderink I, Kieft S, Wilgenburg J (2010) Koken met VENOM : de bereiding van een verkeersprognosemodel voor de Metropoolregio Amsterdam. In: Bijdrage aan het Colloquium Vervoersplanologisch Speurwerk, Roermond

Knoppers P, Muller T (1995) Optimized Transfer Opportunities in Public Transport. Transportation Science 29(1):101-105, DOI 10.1287/trsc.29.1.101

Van Eck G (2011) Dynamische toedeling en toetsing van grootschalige multimodale vervoerssystemen. In: Bijdrage aan het Colloquium Vervoersplanologisch Speurwerk, Antwerpen 


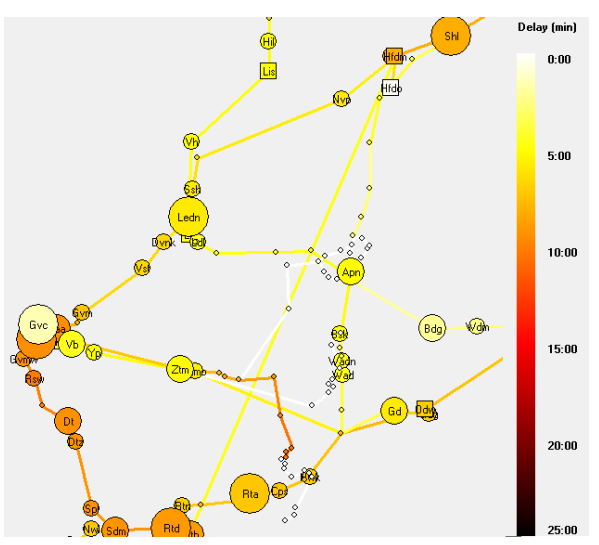

Fig. 4 Vehicle delays if all connections cancelled

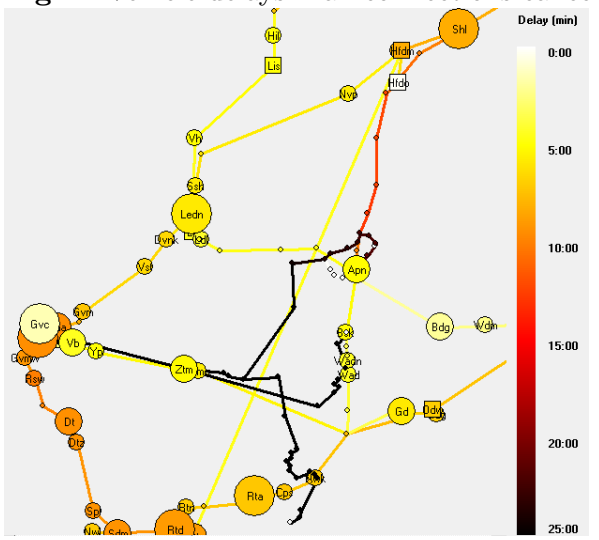

Fig. 5 Passenger delays if all connections cancelled

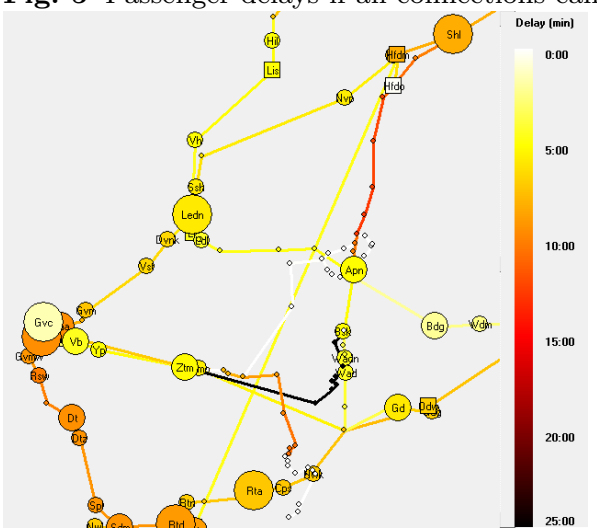

Fig. 6 Passenger delays in case of optimal control 